\title{
Customized Frozen Embryo Transfer after Identification of the Receptivity Window with a Transcriptomic Approach Improves the Implantation and Live Birth Rates in Patients with Repeated Implantation Failure
}

\author{
Delphine Haouzi ${ }^{1,2,3}$ (D) Frida Entezami $^{1,4,5} \cdot$ Antoine Torre $^{6} \cdot$ Charlène Innocenti $^{1,2,3} \cdot$ Yannick Antoine $^{1,2}$. \\ Charlotte Mauries $^{3}$ - Claire Vincens ${ }^{3}$ - Sophie Bringer-Deutsch ${ }^{3}$. Anna Gala ${ }^{1,3} \cdot$ Alice Ferrieres-HOA $^{1,3} \cdot$ Jeanine Ohl $^{7}$. \\ Beatriz Gonzalez Marti ${ }^{4,5}$. Sophie Brouillet ${ }^{1,2,3,8} \cdot$ Samir Hamamah $^{1,2,3}$
}

Received: 7 February 2020 / Revised: 4 June 2020 / Accepted: 30 June 2020 / Published online: 28 July 2020

(C) The Author(s) 2020

\begin{abstract}
The aim of this prospective study was to evaluate outcome benefits expected in repeated implantation failure (RIF) patients $(n=$ 217) after customized embryo transfer based upon identification of the receptivity window by transcriptomic approach using the Win-Test. In this test, the expression of 11 endometrial genes known to be predictive of endometrial receptivity is assessed by RT-PCR in biopsies collected during the implantation window (6-9 days after the spontaneous luteinizing hormone surge during natural cycles, 5-9 days after progesterone administration during hormone replacement therapy cycles). Then, patients underwent either customized embryo transfer (cET, $n=157$ patients) according to the Win-Test results or embryo transfer according to the classical procedure (control group, $n=60$ ). Pregnancy and live birth rates were compared in the two groups. The Win-Test showed that in $78.5 \%$ of women, the receptivity window lasted less than $48 \mathrm{~h}$, although it could be shorter $(<24 \mathrm{~h}$, $9.5 \%)$ or longer $(>48 \mathrm{~h}, 12 \%)$. This highlighted that only in $20 \%$ of patients with RIF the endometrium would have been receptive if the classical embryo transfer protocol was followed. In the other $80 \%$ of patients, the receptivity window was delayed by 1-3 days relative to the classical timing. This suggests that implantation failure could be linked to inadequate timing of embryo transfer. In agreement, both implantation $(22.7 \%$ vs. $7.2 \%)$ and live birth rates per patient $(31.8 \%$ vs. $8.3 \%)$ were significantly higher in the cET group than in the control group. cET on the basis of the Win-Test results could be proposed to improve pregnancy and live birth rates.
\end{abstract}

ClinicalTrials.gov ID: NCT04192396; December 5, 2019, retrospectively registered.

Keywords Transcriptome $\cdot$ Win-Test $\cdot$ Implantation window $\cdot$ Receptivity window

Electronic supplementary material The online version of this article (https://doi.org/10.1007/s43032-020-00252-0) contains supplementary material, which is available to authorized users.

Delphine Haouzi

delphine.haouzi@inserm.fr

1 Univ Montpellier, INSERM U1203, EmbryoPluripotency, Montpellier, France

2 IRMB, Univ Montpellier, INSERM, Montpellier, France

3 CHU Montpellier, ART/PGD Department, Arnaud de Villeneuve Hospital, Montpellier, France

4 ART Department, American Hospital of Paris, Neuilly-Sur-Seine, France
5 Laboratoire Eylau-UNILABS-La Muette, Clinique de La Muette-Ramsay-Générale de Santé, Paris, France

6 Division of Child Health, Obstetrics \& Gynaecology Department, University of Nottingham, Nottingham, UK

7 Department of reproductive medicine, CMCO, Schiltigheim, France

8 Univ Grenoble-Alpes, INSERM U1036, Commissariat à l'énergie atomique et aux énergies alternatives (CEA), Institut de Biosciences et Biotechnologies de Grenoble (BIG), Laboratoire Biologie du Cancer et de l'Infection (BCI), 38000 Grenoble, France 


\section{Introduction}

Infertility is a major public health problem that affects more than one in six couples of childbearing age. According to a recent French national perinatal survey and the French epidemiological observatory for fertility, almost $40 \%$ of couples do not conceive after 1 year of regular sexual intercourses without contraception $[1,2]$. Moreover, the average live birth rate after assisted reproductive technology (ART), irrespective of age and indication, is low $(<20 \%)$ [3]. Despite the many ART advances, embryo implantation rate remains problematically low. Implantation failure is mainly correlated with embryo competency and lack of uterine receptivity leading to altered embryo-endometrium synchronization. Several non-invasive parameters have been proposed to assess endometrial receptivity and the "implantation window" timing, such as endometrium thickness, endometrial morphology by ultrasound assessment, and endometrial and sub-endometrial blood flow measure by Doppler ultrasound $[4,5]$. However, these approaches have given controversial results and their positive predictive value for endometrial receptivity evaluation is low [5,6]. Yet, it is crucial to exactly determine the endometrial receptivity occurrence and duration. Transcriptomic and proteomic approaches also have been used to define molecular signatures and identify specific biomarkers of human endometrial receptivity (comprehensive review in [7]). Yet, very few molecular diagnostic tools are available to characterize the implantation window [8,9]. Using our transcriptomic data, we previously identified a set of genes (BCL2L10, CD68, TRPC4, SORCS1, FST, KRT18, LAMB3, MFAP5, ANGPTL1, PROK1, and $C 2 C D 4 B$ ) that are overexpressed in the endometrium during the implantation window and that seem to be relevant candidate biomarkers of human endometrial receptivity [7, 10-14]. After validation of these transcriptomic results by reverse transcription-quantitative PCR (RTqPCR), we tested these candidate endometrial receptivity biomarkers in fertile patients and in an ex vivo model (i.e., stromal and epithelial endometrium cells) $[10,14$, 15]. We then developed an innovative test based on the quantification by RT-PCR of 11 of these genes in endometrium biopsies that we called Win-Test for Window Implantation Test [16]. The strength and robustness of the Win-Test have been extensively reviewed in [7]. However, the Win-Test clinical benefit in terms of pregnancy and live birth rates has never been established. Therefore, the aim of this prospective interventional multicenter study was to determine the ART outcomes (pregnancy and live birth rates) after frozen embryo transfer according to the Win-Test results compared with the usual procedure.

\section{Materials and Methods}

\section{Study Design and Sample Collection}

Between January 2015 and June 2018, 217 women (mean \pm $\mathrm{SD}$, age $37.04 \pm 4.4$ years) were recruited after signature of the written informed consent and approval by the IRB of the Montpellier University Hospital. All recruited women had a history of repeated implantation failure (RIF), according to the definition by Polanski et al. [17] (mean \pm SD, number of previous failed attempts $4.58 \pm 2.15$ ), after fresh and/or frozenthawed embryo transfer. This sample included also patients who benefited from oocyte/embryo donation $(n=41)$. Patients were referred for female (44.9\%), male (23.4\%), idiopathic (21\%), and mixed (10.7\%) infertility. All underwent a classical infertility evaluation that included transvaginal sonography and uterine cavity assessment by hysteroscopy. In addition, the following data were recorded after each implantation failure (thrombophilia, coagulation factors, immunologic response, and thyroid function). All patients were scheduled for cryopreserved embryo transfer during a hormone replacement therapy (HRT) cycle with ( $n=44$ patients) or without $(n=111)$ $\mathrm{GnRH}$ analogue (GnRHa), or during a natural cycle $(n=62)$.

All patients underwent endometrial receptivity estimation with the Win-Test during the theoretical implantation window between days 5 and 9 after progesterone treatment start $(\mathrm{Pg}+5$ to $\mathrm{Pg}+9$ ) or between day 6 and day 9 after luteinizing hormone surge $(\mathrm{LH}+6$ to $\mathrm{LH}+9)[10,15]$. Biopsy was rinsed in PBS, then placed in a cryotube containing lysis buffer (RLT; Qiagen), and quickly frozen at minimum $-80^{\circ} \mathrm{C}$ (dry ice or liquid nitrogen) until shipment in dry ice to our Montpellier ART center.

According to each center protocol, the HRT regimen involved estradiol administration (fixed dose of 4 to $6 \mathrm{mg}$ /day of estrogen; or a progressively increasing dose from $2 \mathrm{mg}$ to a maximum of $6 \mathrm{mg}$ daily, changing dose every 4 days) through the oral route and/or transdermal patch starting from day 1 or 2 of the menstrual cycle until progesterone administration. The final endometrial maturation was obtained with 600 to $800 \mathrm{mg}$ progesterone per day. Some patients received a subcutaneous/ intramuscular injection of triptorelin $(3 \mathrm{mg})$ at day 21 of the previous menstrual cycle. The endometrium ultrasound pattern and thickness were assessed between days 12 and 14 after menses and progesterone was administered when endometrium showed a trilaminar pattern and a thickness $>7 \mathrm{~mm}$. The number of progesterone treatment days was calculated according to the daily progesterone dose (1 day of complete dose corresponded to $\mathrm{Pg}+1$ ) (Fig. 1).

For patients on natural cycle, estradiol, progesterone, and LH were quantified in serum to determine the LH surge. Specifically, the exact time of spontaneous ovulation was determined retrospectively on the basis of (1) baseline serum $\mathrm{LH}$ and progesterone concentration at days 1-3 of the cycle; (2) serum estradiol, progesterone $(<1.5 \mathrm{ng} / \mathrm{ml})$, and $\mathrm{LH}$ 


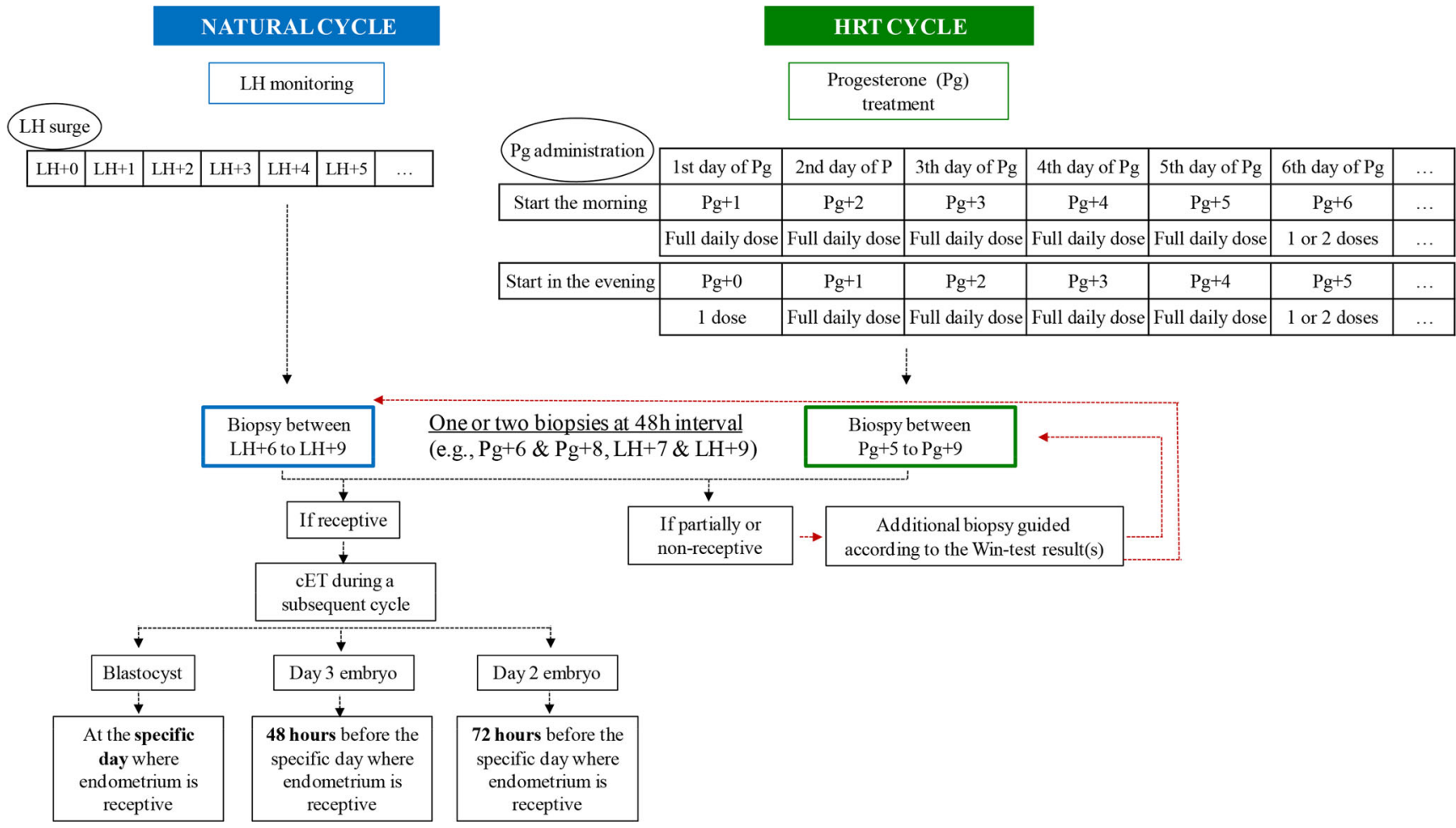

Fig. 1 Outline of the Win-Test procedure. HRT, hormone replacement therapy; LH, luteinizing hormone; Pg, progesterone; cET, customized embryo transfer

concentration from day 10 of the cycle to the LH surge (up to daily quantification, if required; serum LH level increase by more than threefold compared with the baseline value); and (3) serum progesterone increase to more than $1.5 \mathrm{ng} / \mathrm{ml}$ after the LH surge. The day of LH surge was considered as day LH +0 (Fig. 1)

\section{The Win-Test}

The 11 genes involved in human endometrial receptivity included in the Win-Test were selected by comparing the gene expression profiles of receptive $(\mathrm{LH}+7, n=31)$ and prereceptive $(\mathrm{LH}+2, n=31)$ endometrium obtained in our previous transcriptomic analysis [10]. Specifically, the Significant Analysis of Microarrays (SAM; Stanford University, USA [18]) and $t$ test were used to select genes with an absolute fold-change $>2$ and a false discovery rate $<$ 0.05 . Concomitantly, class prediction applied to microarray experiments was used to identify biomarkers that are putatively involved in endometrial receptivity [10, 19]. The 11 most upregulated genes identified by both approaches were included in the Win-Test [7, 10-13]. Each test includes a receptive endometrium $(\mathrm{LH}+7)$ and a pre-receptive endometrium $(\mathrm{LH}+2)$ sample as positive and negative control, respectively. Each gene of the Win-Test must be overexpressed compared with a pre-receptive endometrium to define the endometrium as "receptive." As the microarray data clearly showed that the expression of the 11 genes might vary among patients, threshold values of their mean of expression were defined to take into account this inter-patient variability. Accordingly, an endometrium is defined as "receptive" when the mean expression of the 11 genes is $\geq 70 \%$, "partially receptive" when between 50 and $70 \%$, and "non-receptive" when $<50 \%$ of the expression level of the positive control which is meant to be $100 \%$ and the negative control $<15 \%$. The Win-Test results are provided within 5 days post-reception of biopsies.

The Win-Test performance to predict pregnancy outcome using receiver operating characteristics analysis was evaluated in the first RIF patients who underwent customized embryo transfer (cET) as described in [20] (Supplementary Fig. S1).

\section{Customized Frozen Embryo Replacement According to the Win-Test Results}

Our strategy consisted in performing cET of blastocysts based on the endometrium receptivity day identified using the WinTest. Therefore, frozen day 2 or day 3 embryos were transferred 72 or $48 \mathrm{~h}$ before this day, respectively. When the endometrial sample was defined as non-receptive or partially receptive by the Win-Test, a second evaluation was performed subsequently according to the first Win-Test result (Fig. 1).

Positive pregnancy test was defined as a positive $\beta$-hCG serum concentration followed by at least two increasing $\beta$ hCG values at 48 -h interval, to exclude early biochemical 
pregnancies. Clinical pregnancy was defined by the ultrasound visualization of a gestational sac with embryo heartbeat. The implantation rate (i.e., the number of observed gestational sacs relative to the total number of transferred embryos) and live birth rate (i.e., the birth of at least one live baby after more than 24 weeks of amenorrhea) were also recorded after cET according to the Win-Test results. In the control group, embryo transfer was performed as usually routine protocol (i.e., at $\mathrm{Pg}+5 / \mathrm{Pg}+6$ for HRT cycles and at $\mathrm{LH}+6$ / $\mathrm{LH}+7$ for natural cycles).

\section{Quantitative RT-PCR Analyses}

For the Win-Test, $0.5 \mu \mathrm{g}$ RNA from each endometrial sample was used for RT-qPCR analysis according to the manufacturer's recommendations (Applied Biosystems, Villebonsur Yvette, France), as previously described [15, 16]. For qPCR, $2 \mu \mathrm{l}$ of first strand DNA (1:5 dilution) was added to a $10-\mu 1$ reaction mixture containing $0.25 \mu \mathrm{M}$ of each primer and $5 \mu \mathrm{l}$ of 2× LightCycler 480 SYBR Green I Master mix (Roche, Mannheim, Germany). DNA was amplified for 45 cycles with an annealing temperature of $63^{\circ} \mathrm{C}$ using the Light Cycler 480 detection system (Roche). Gene expression values were normalized to the expression of three housekeeping genes, hypoxanthine phosphoribosyltransferase 1 (HPRT1), glyceraldehyde-3-phosphate dehydrogenase $(G A P D H)$, and phosphoglycerate kinase 1 ( $P G K 1)$ using the following formula: $E_{\text {tested gene }} \Delta \mathrm{Ct} / E_{\text {housekeeping gene }} \Delta \mathrm{Ct}\left(E=10^{-1 / \text { slope }}\right)$, $\Delta \mathrm{Ct}=\mathrm{Ct}$ control $-\mathrm{Ct}$ unknown, where $E$ corresponds to the PCR reaction efficiency. The $E$ value was obtained using a standard curve that varies in function of the used primers. One receptive endometrium sample from a patient in natural cycle $(\mathrm{LH}+7)$ was used as positive control and a non-receptive sample $(\mathrm{LH}+2)$ as negative control. Each sample was analyzed in duplicate and multiple water blanks were included.

\section{Statistical Analyses}

Statistical analyses were performed with the GraphPad Prism 8 software. Data are expressed as the mean \pm SD and differences between groups were considered significant when the Student's $t$ test gave a $P$ value $<0.05$. ROC curve analysis was performed with the MedCalc software, according to the methodology described by DeLong et al. [21].

\section{Results}

\section{The Receptivity Window Duration Is Patient Dependent}

In total, 419 endometrial biopsies from 217 patients with RIF were analyzed: $51.1 \%$ of patients $(n=111)$ were in HRT cycles, $20.3 \%(n=44)$ in HRT with GnRHa, and $28.6 \%$ in natural cycles $(n=62)$. Most patients underwent one $(35 \%)$ or two consecutive endometrial biopsies at 48-h interval (53\%). Only $12 \%$ of patients needed three or more biopsies to identify the receptivity window (Fig. 2a). No more than two endometrial biopsies were performed during a single cycle, at $\mathrm{Pg}+6$ and $\mathrm{Pg}+8$, or $\mathrm{LH}+7$ and $\mathrm{LH}+9$ under HRT or natural cycles, respectively. For patients requiring more than two endometrial biopsies, at least one was performed during a subsequent cycle with the same treatment and biopsy timing was guided according to the first Win-Test result (e.g., if partially receptive at $\mathrm{LH}+7$ and non-receptive at $\mathrm{LH}+9$ the additional biopsy is recommended at $\mathrm{LH}+8$ ). Overall, the number of biopsies per patient was not significantly different between patients in natural and HRT cycles.

Among the patients with one endometrial biopsy $(n=75)$, the receptivity window was identified in $73(97 \%)$. In the group of patients with two endometrial biopsies at 48-h interval $(n=115)$, the receptivity window was identified in 111 (97\%) (Fig. 2a). In addition, the endometrium was considered receptive only in one of the two biopsies in 98 women (88\%) and in both in 13 patients (12\%). In the group of patients with three endometrial biopsies $(n=27)$, the receptivity window was identified in 24 patients (89\%) (Fig. 2a).

In the group of patients who underwent two or more endometrial biopsies with successful identification of the receptivity window $(n=135)$, the receptivity window lasted $24-48 \mathrm{~h}$ in 106 patients (78.5\%). Its duration was longer than $48 \mathrm{~h}$ in 16 patients $(12 \%)$ and shorter than $24 \mathrm{~h}$ in 13 patients $(9.5 \%)$ (Fig. 2b).

a

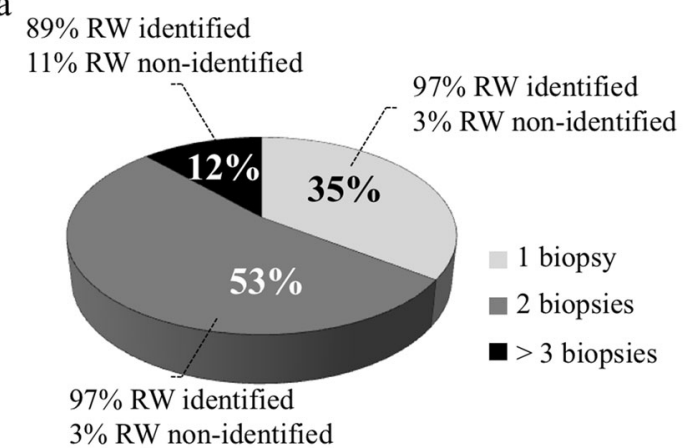

b

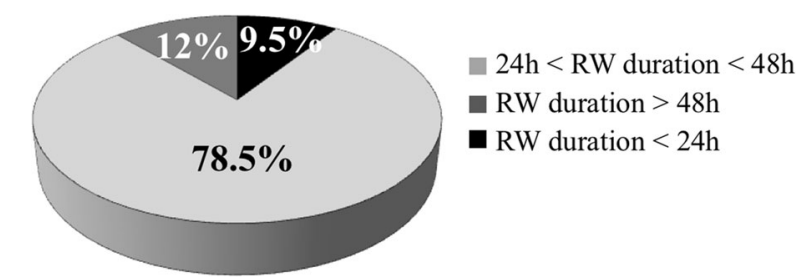

Fig. 2 a Percentage of patients with RIF in whom the endometrial receptivity window (RW) was or was not identified in function of the number of endometrial biopsy performed. $\mathbf{b} \mathrm{RW}$ duration in patients with RIF who had at least two endometrial biopsies 
Whatever the receptivity window duration, the analysis of the endometrial receptivity status at different time points within the same cycle or in subsequent cycles (under the same condition/treatment) in the same patient showed that endometrial receptivity acquisition was a progressive process, as revealed by the mean expression levels of the Win-Test genes, during both natural and $\mathrm{HRT} \pm \mathrm{GnRHa}$ cycles with a tendency for a more progressive process under HRT. Conversely, the implantation window closure occurred rapidly, within $24 \mathrm{~h}$ after its detection (Supplementary Fig. S2).

\section{The Appearance of the Receptivity Window Is Patient Dependent}

Among the 62 patients in natural cycle, the Win-Test was performed at $\mathrm{LH}+6 / \mathrm{LH}+7$ in 46 patients. At this specific time point, only $30.5 \%(14 / 46)$ were receptive, while the others were either partially receptive $(28 \%, 13 / 46)$ or nonreceptive $(41.5 \%, 19 / 46)$ (Fig. 3a). In total, the receptivity window was identified in 57/62 women (92\%), and was mainly at $\mathrm{LH}+8(42 \%, 24 / 57)$, followed by $\mathrm{LH}+6 / \mathrm{LH}+7$ $(24.5 \%, 14 / 57)$ and $\mathrm{LH}+9(33.5 \%, 19 / 57)$ (Fig. 2b). Among the 111 patients on HRT, 61 patients were evaluated at $\mathrm{Pg}+5 /$ $\mathrm{Pg}+6$, and $25 \%$ were receptive $(15 / 61), 31 \%$ partially receptive $(19 / 61)$, and $44 \%$ non-receptive $(27 / 61)$. The receptivity window could be identified in $108 / 111$ patients $(97 \%)$ on HRT, mainly at $\mathrm{Pg}+7(30.5 \%, 33 / 108)$ and $\mathrm{Pg}+8(46 \%$, 50/108). For the remaining patients, endometrium was receptive at $\mathrm{Pg}+5 / \mathrm{Pg}+6(14 \%, 15 / 108)$ and $\mathrm{Pg}+9(9 \%, 10 / 108)$ (Fig. 3b).

Among the 44 patients on HRT with GnRHa, 39 patients were evaluated at $\mathrm{Pg}+5 / \mathrm{Pg}+6$. Endometrium was receptive in $26 / 39(67 \%)$, partially receptive in $6 / 39(15 \%)$, and nonreceptive in $7 / 39$ (18\%) (Fig. 3a). In total, the receptivity window could be identified in $41 / 44$ patients, mostly at $\mathrm{Pg}+8$ (63\%; 26/41), followed by $\mathrm{Pg}+5 / \mathrm{Pg}+6$ (17\%; 7/41), Pg + 7 (4\%; 4/41), and $\mathrm{Pg}+9$ (4\%; 4/41) (Fig. 3b).

\section{Pregnancy Outcome According to the Win-Test Strategy in Patients with RIF}

To evaluate the clinical suitability of the Win-Test, 157 patients underwent embryo transfer according to the Win-Test results (cET group) and 60 (control group) according to the a

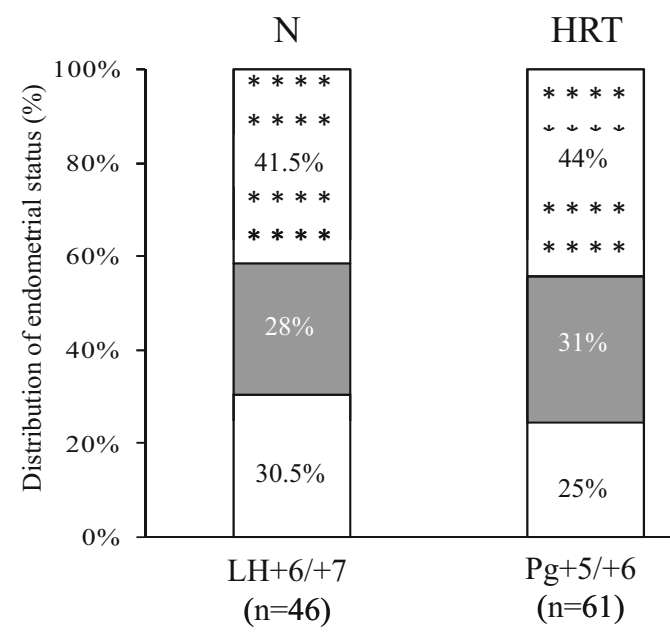

$\mathrm{HRT}+\mathrm{GnRHa}$

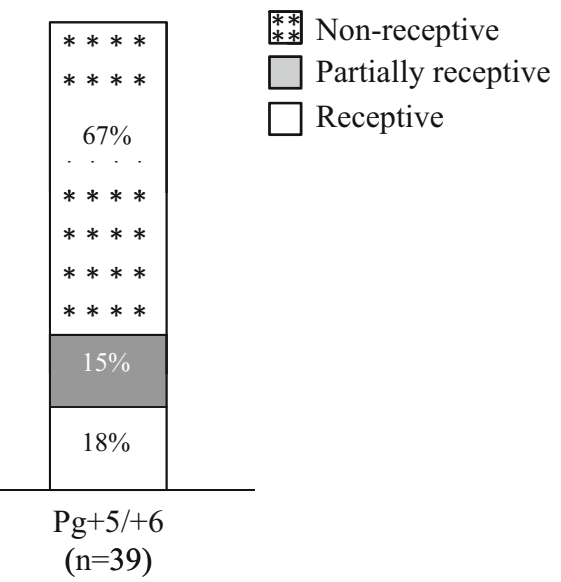

b

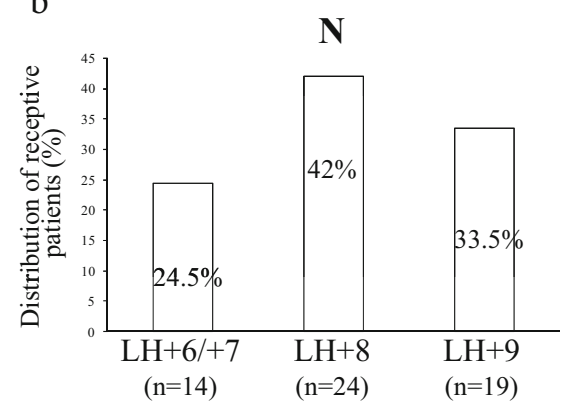

HRT

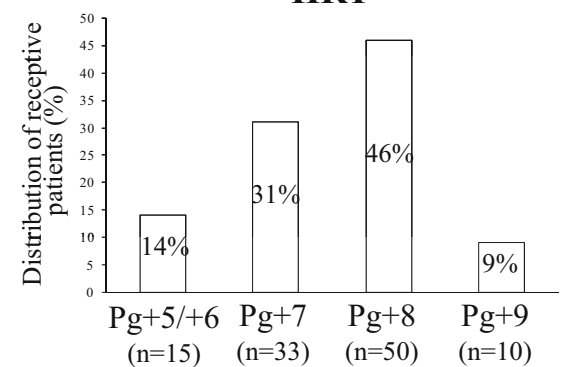

HRT + GnRHa

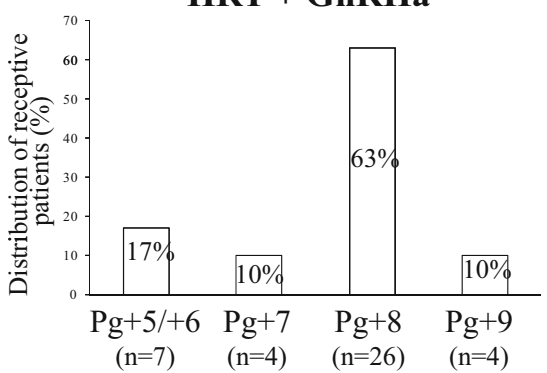

Fig. 3 (a) Endometrial receptivity status according to the Win-Test performed at $\mathrm{LH}+6 / \mathrm{LH}+7$ (natural cycles) and at $\mathrm{Pg}+5 / \mathrm{Pg}+6$ (HRT cycles with or without GnRHa). (b) Percentage of patients with receptive endometrium (Win-Test) at different time points during natural and HRT cycles with or without GnRHa. GnRHa, GnRH analogue; HRT, hormone replacement therapy; LH, luteinizing hormone; N, natural cycle; Pg, progesterone 
classical protocol. The mean age (mean $\pm \mathrm{SD}, 37.2 \pm 4.3$ vs. $36.7 \pm 4.5$ years, $p=0.47$ ), number of previous failed attempts (mean $\pm \mathrm{SD}, 4.4 \pm 1.9$ vs. $4.9 \pm 2.6, p=0.18$ ), number of previous non-implanted embryos (mean $\pm \mathrm{SD}, 6.4 \pm 3.6$ vs. $7.5 \pm$ $4.2, p=0.06)$, and infertility etiology (11\% vs. $10.2 \%$ with both female and male infertility, $p=0.9 ; 24.5 \%$ vs. $20.3 \%$ with male infertility, $p=0.6 ; 45.2 \%$ vs. $44.1 \%$ with female infertility, $p=0.9 ; 19.4 \%$ vs. $25.4 \%$ with idiopathic infertility, $p=0.35$ ) were comparable in the cET and control groups (Table 1). The proportion of patients who benefited from oocyte/embryo donation also was similar between groups (21.3\% in the cET and $13.6 \%$ in the control group, $p=$ $0.25)$. Moreover, the proportion of patients with delayed receptivity window (i.e., after $\mathrm{LH}+6 / \mathrm{LH}+7$ or $\mathrm{Pg}+5 / \mathrm{Pg}+6$ ) was similar between groups $(81.9 \%$ vs. $86.6 \%$ in the cET and control group, $p=0.64)$. Specifically, among the 46 patients in a natural cycle of the cET group, endometrium was receptive at $\mathrm{LH}+8$ in $48 \%$, at $\mathrm{LH}+6 / \mathrm{LH}+7$ in $22 \%$, and at $\mathrm{LH}+9$ in $30 \%$ (Fig. 4). Among the 73 patients on HRT who underwent $\mathrm{cET}$, endometrium was receptive at $\mathrm{Pg}+7(29 \%)$ and $\mathrm{Pg}+8$ (54\%), followed by $\mathrm{Pg}+5 / \mathrm{Pg}+6(10 \%)$ and $\mathrm{Pg}+$ $9(7 \%)$. Among the 38 patients on HRT with GnRHa who underwent $\mathrm{cET}$, endometrium was receptive at $\mathrm{Pg}+8$ in $63 \%$, at $\mathrm{Pg}+5 / \mathrm{Pg}+6$ in $13 \%$, at $\mathrm{Pg}+7$ in $13 \%$, and at $\mathrm{Pg}+$ 9 in $11 \%$ (Fig. 4).

The implantation rate per cycle $(22.7 \%$ vs. $7.2 \%, p=$ $0.0001)$ was significantly higher in the cET than in the control group (Table 2). In the cET group, a total of 277 embryos were transferred (63 gestational sacs/277 transferred embryos), resulting in 50 live births. In the control group, 125 embryos were transferred (9 gestational sacs/125 transferred embryos), resulting in 5 live births $(p=0.0002)$ (Table 2). Similarly, the positive $\beta$-hCG and clinical pregnancy rates per patient were higher in the cET than in the control group (44.6\% vs. $16.7 \%$, $p=0.0001$; and $38.8 \%$ vs. $15 \%, p=0.0006)$. The live birth rate per patient was $31.8 \%$ in the $\mathrm{cET}$ and $8.3 \%$ in the control group ( $p=0.0002)$, despite the fact that the mean ( \pm SD) number of transferred embryos per cycle was smaller in the cET than in the control group $(1.4 \pm 0.5$ vs. $1.6 \pm 0.7, p=0.04)$ (Table 1). At the time of data collection, seven pregnancies in the cET group (4.5\%) were still ongoing. Therefore, the live birth rate was underestimated for the cET group. After the first attempt, the implantation and live birth rates after cET were $24.8 \%$ (56 gestational sacs/226 embryos transferred) and $31.2 \%$, respectively. The pregnancy outcomes per cycle according to the stage of the transferred embryo (cleavage stage embryos vs. blastocysts) are provided in Supplementary Table S1. Whatever the embryo stage, the pregnancy rate per cycle was significantly higher in the cET compared with the control group.

\section{Discussion}

The Win-Test allowed identification of the endometrial receptivity window within the implantation window. Whatever the cycle type (natural, HRT, HRT with GnRHa), the receptivity window duration was longer than $24 \mathrm{~h}$ and shorter than $48 \mathrm{~h}$ in $78.5 \%$ of patients. Duration was longer than $48 \mathrm{~h}$ in $12 \%$ and shorter than $24 \mathrm{~h}$ in $9.5 \%$ of patients. This can explain why in a sub-group of patients (12\%) more than two endometrial biopsies were necessary to identify the receptivity window. No more than two endometrial biopsies were performed during the same cycle, mainly at $48 \mathrm{~h}$ of interval, because local

Table 1 Patients' characteristics

\begin{tabular}{llll}
\hline & cET & Controls & $p$ value \\
\hline Number of patients & 157 & 60 & \\
Age (years) & $37.2 \pm 4.3$ & $36.7 \pm 4.5$ & $\mathrm{~ns}$ \\
Number of previous failed attempts (FET, FTET) & $4.4 \pm 1.9$ & $4.9 \pm 2.6$ & $\mathrm{~ns}$ \\
Number of previous non-implanted embryos (FET, FTET) & $6.4 \pm 3.6$ & $7.5 \pm 4.2$ & $\mathrm{~ns}$ \\
Infertility etiology (\% of patients): & & & \\
$\quad$ Both (female and male) & 11 & 10.2 & $\mathrm{~ns}$ \\
Female & 45.2 & 44.1 & $\mathrm{~ns}$ \\
$\quad$ Male & 24.4 & 20.3 & $\mathrm{~ns}$ \\
$\quad$ Idiopathic & 19.4 & 25.4 & $\mathrm{~ns}$ \\
Endometrial thickness (mm) & $8.9 \pm 1.6$ & $8.6 \pm 1.2$ & $\mathrm{~ns}$ \\
Number of transferred embryos (per cycle) & $1.4 \pm 0.5$ & $1.6 \pm 0.7$ & 0.04 \\
Cleavage stage embryos transferred (\% of patients) & 50.6 & 59.5 & $\mathrm{~ns}$ \\
Blastocysts transferred $\%$ of patients) & 49.4 & 40.5 & $\mathrm{~ns}$ \\
\hline
\end{tabular}

Data are expressed as the mean $\pm \mathrm{SD}$

$c E T$ customized embryo transfer, FET fresh embryo transfer, FTET frozen-thawed embryo transfer, ns nonsignificant 

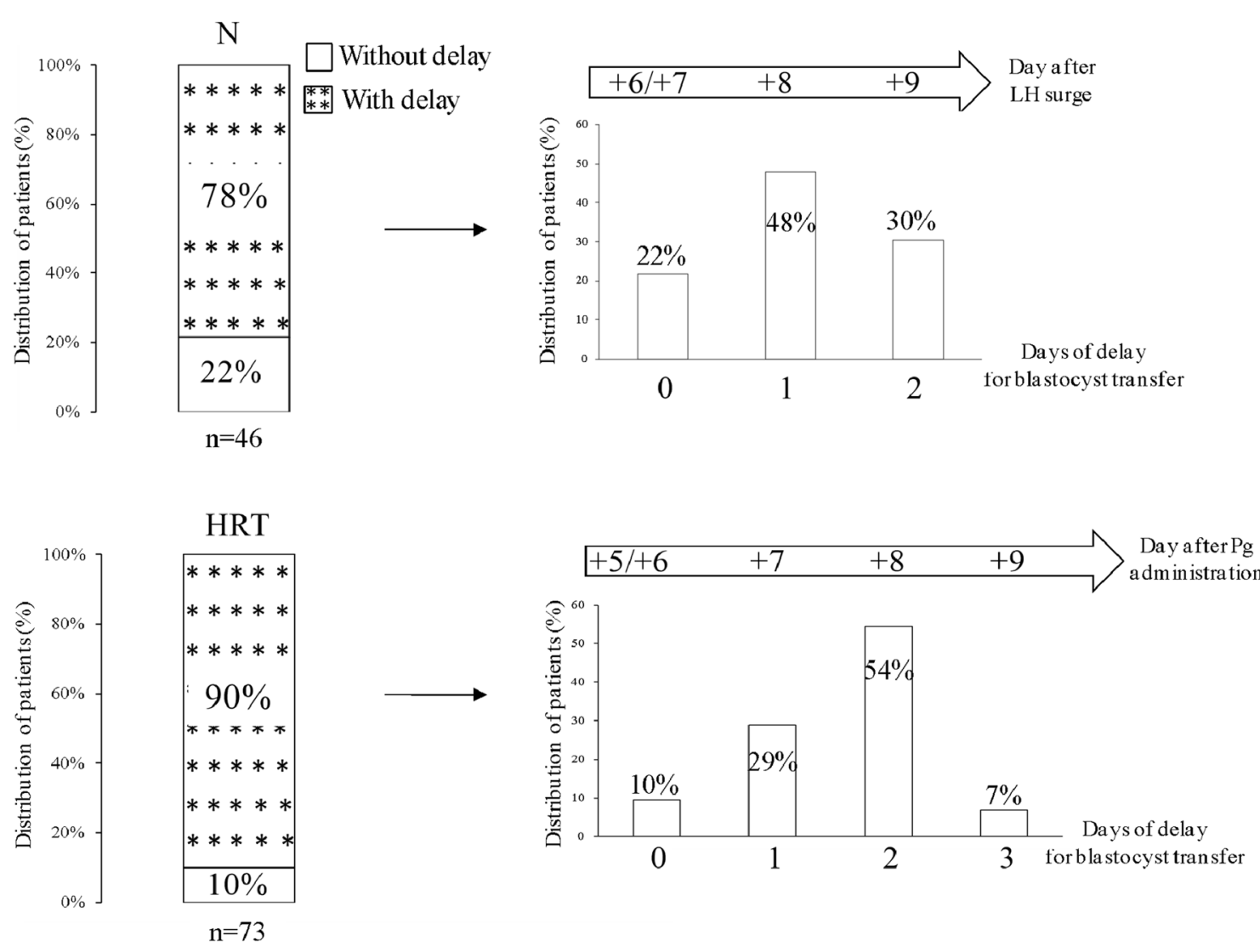

\section{$\mathrm{HRT}+\mathrm{GnRHa}$}

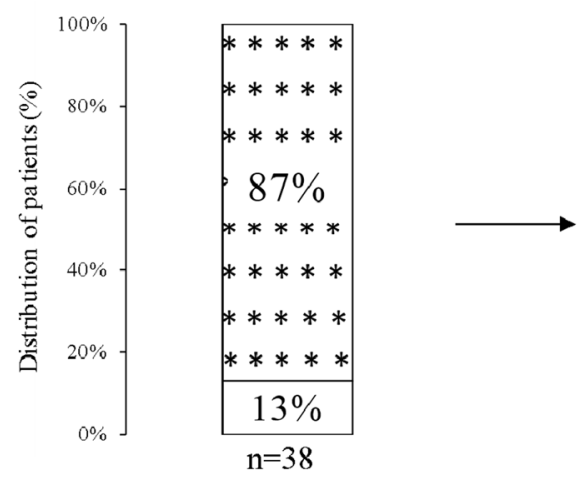

Fig. 4 Receptivity window timing in the 157 patients with RIF ( $n=46$ in natural cycles; $n=73$ in HRT cycles; $n=38$ in HRT with GnRHa cycles) who underwent cET according to the Win-Test results. The receptivity window was considered delayed relative to the classical embryo transfer

injury caused by consecutive biopsies can affect the endometrial transcriptomic profile [22]. Nevertheless, none of genes that are affected by endometrial biopsy-induced local injury is included in the Win-Test gene panel. Previous studies suggested that the implantation window may last $48 \mathrm{~h}$ [23], 2 to 4 days [24], 4 days [25], or 3 to 6 days [26]. Here, we showed that the receptivity window lasts about 2 days in most patients $(\sim 78 \%)$. This information is crucial and must be taken into account for cET of cryopreserved embryos. Our data from patients with three or more endometrial biopsies during at strategy for natural and HRT cycles. B1, blastocyst; cET, customized embryo transfer; GnRHa, GnRH analogue; LH, luteinizing hormone; $\mathrm{Pg}$, progesterone; RIF, repeated implantation failure

least two identical cycles suggest that the acquisition of the endometrial receptivity phenotype is a progressive process during both HRT and natural cycles. This notion is reinforced by the fact that the decidualization, essential for the acquisition of the endometrial receptivity phenotype, is also a progressive process that starts during the postovulatory phase (early-secretory phase) and culminates during the midsecretory phase [14]. Conversely, the implantation window closes very rapidly, within $24 \mathrm{~h}$ after the occurrence of the receptivity window. In addition, we found that the receptive 
Table 2 Pregnancy outcome in patients with RIF after cET according to the Win-Test results and after the classical procedure (controls)

\begin{tabular}{llll}
\hline & cET & Controls & $p$ value \\
\hline Pregnancy outcome/patient & & & \\
Number of patients & 157 & 60 & \\
Pregnancy rate $(\beta$-hCG+) (\%) & $70(44.6)$ & $10(16.7)$ & 0.0001 \\
Clinical pregnancy rate (\%) & $61(38.8)$ & $9(15)$ & 0.0006 \\
Ongoing pregnancy rate (\%) & $57(36.3)$ & $5(8.3)$ & 0.00002 \\
Live birth rate (\%) & $50(31.8)$ & $5(8.3)$ & 0.0002 \\
Pregnancy outcome/cycle & & & \\
Number of cycles & 195 & 79 & \\
Pregnancy rate ( $\beta$-hCG+) (\%) & $75(38.5)$ & $10(12.7)$ & 0.00002 \\
Clinical pregnancy rate (\%) & $61(31.3)$ & $9(11.4)$ & 0.0004 \\
Ongoing pregnancy rate (\%) & $57(29.2)$ & $5(6.3)$ & 0.00001 \\
Live birth rate (\%) & $50(25.6)$ & $5(6.3)$ & 0.0002 \\
Implantation rate $(\%)$ & $63 / 277(22.7)$ & $9 / 125(7.2)$ & 0.0001 \\
\hline
\end{tabular}

Data are expressed as the mean $\pm \mathrm{SD}$. The number of patients and cycles are indicated, and the percentage of patients or cycles is between brackets $c E T$ customized embryo transfer

status timing is specific to each patient. According to the usual strategy for frozen-thawed blastocyst transfer during natural cycles, endometrium receptivity is considered to occur at $\mathrm{LH}+6 / \mathrm{LH}+7$ [27-29]. Here, we showed that only $30 \%$ of the 46 patients with RIF who were screened with the Win-Test at these specific times had a receptive endometrium. In the 57 patients in a natural cycle in whom endometrium receptivity could be identified, the majority had a receptive endometrium at $\mathrm{LH}+8(42 \%)$ and $\mathrm{LH}+9(33.5 \%)$, and only $24.5 \%$ at $\mathrm{LH}+6 / \mathrm{LH}+7$. During HRT cycles, blastocysts are usually transferred at $\mathrm{Pg}+5 / \mathrm{Pg}+6[28-33]$. At this time, only $25 \%$ of patients with RIF were identified as receptive, whereas $31 \%$ were receptive at $\mathrm{Pg}+7$ and $46 \%$ at $\mathrm{Pg}+8$. The others were receptive at $\mathrm{Pg}+5 / \mathrm{Pg}+6(14 \%)$ and at $\mathrm{Pg}+9(9 \%)$.

These findings indicate that in most of our patients with RIF, the receptivity window was delayed by 1 to 2 days in natural cycles, and between 1 and 3 days during HRT, with or without GnRHa, reinforcing the hypothesis that the acquisition of the endometrial receptivity phenotype is slower in HRT than in natural cycles. This delay is consistent with previous reports suggesting that the endometrium of patients with RIF tends to be pre-receptive at $\mathrm{Pg}+5$ [34-36]. At $\mathrm{Pg}+5 /$ $\mathrm{Pg}+6$, the receptivity window was delayed in $\sim 80 \%$ of our patients with RIF compared with $26 \%$ ( $n=85$ patients with RIF) and 47\% ( $n=62$ patients with RIF) in the studies by Ruiz-Alonso et al. [34] and Tan et al. [36], respectively. In these two studies, endometrial receptivity was tested at $\mathrm{Pg}+5$ using a different assay, and RIF was defined in a different manner in the study by Tan et al. [36]. Conversely and differently from other reports [34], we never observed early endometrial receptivity occurrence during HRT and natural cycles.
Our results are in accordance with the study by Bassil et al. [37] showing that only $35 \%$ of 41 patients with $0-2$ previous failed embryo transfers were receptive at Pg +5 (HRT cycles). These findings strongly suggest that the delay observed in patients in HRT cycles is a specific feature of HRT protocols and not of patients with RIF, contrary to what was suggested by other authors $[38,39]$. Moreover, we previously reported differences in gene expression profile during the implantation window between HRT and natural cycles [40]. Indeed, in most patients, a minimum time of progesterone treatment seems to be necessary for endometrium maturation and for receptivity acquisition. Indeed, whatever the hormonal treatment, endometrium was receptive after 7 or 8 days of progesterone administration in patients with RIF. These results are consistent with the study by Prapas et al. [25] showing a significant higher pregnancy rates when day 2 embryos (4-6 cells) were transferred at day 4 or 5 after initiation of progesterone compared with day 2 or 3 . In addition, as reported in the present study, endometrium receptivity occurrence time and duration are patient dependent, reinforcing the notion that the identification of the optimal timing for embryo transfer for each patient is essential to optimize ART effectiveness and to achieve a successful pregnancy. Indeed, endometrial receptivity timing varies among patients with similar characteristics (age, infertility etiology) and the same substitutive treatment (similar dose of estrogen and progesterone). Furthermore, some patients in HRT cycles who were evaluated with the Win-Test at more than 1 year of interval showed a similar endometrial profile, suggesting low intra-patient variability (data not shown).

The clinical relevance of the Win-Test was evaluated by performing cET according to the Win-Test results $(n=157$ patients with RIF) or classical embryo transfer $(n=60$ patients with RIF). The two groups were comparable in age, number of previous failed attempts, number of previous non-implanted embryos, and endometrial thickness during the periovulatory period. The fertility outcomes for patients who underwent conventional embryo transfer were consistent with previously published data showing lower pregnancy and implantation rates in women with RIF than without RIF [39]. Comparison of the two groups highlighted significantly higher pregnancy, clinical pregnancy, and live birth rates per patient in the cET than in the control group. Whatever the stage of the transferred embryos (cleavage stage embryos vs. blastocysts), the WinTest strategy improved pregnancy outcomes despite the greater mean number of transferred embryos per cycle in the control group. These findings are very encouraging, and the WinTest interest is currently investigated in a large cohort of patients without RIF.

This was the first prospective study reporting the Win-Test clinical effectiveness as a diagnostic tool to identify the receptivity window and to perform, in a subsequent identical cycle, cET according to the Win-Test results to improve the embryo- 
endometrial synchronization. The other strengths of the present study are the larger cohort of patients with RIF compared with previous studies [34, 37, 38, 41, 42], and the presence of a control arm to compare pregnancy outcomes among RIF patients who did and did not undergo cET after the WinTest. The heterogeneity of protocols (natural and HRT \pm GnRHa cycles) used for endometrial preparation is a limitation. However, the protocol remained the same for each patient during the assessment with the Win-Test and embryo transfer.

\section{Conclusion}

By determining the specific cycle day within the implantation window where endometrium is receptive (i.e., receptivity window), the Win-Test showed that both the occurrence time and duration of the receptivity window are patient dependent during natural and HRT cycles. Moreover, the Win-Test highlighted that implantation failure could be partly due to inadequate timing of embryo transfer, resulting in embryo-endometrial desynchronization. Consequently, cET after the Win-Test to assess the endometrial receptivity status improves implantation, pregnancy, and live birth rates in patients with RIF.

Acknowledgments The authors thank the research direction of the University Hospital of Montpellier, Montpellier University, and Inserm for financial support.

Funding Information This work was partially supported by a grant from the Gedeon Richter Pharmaceutical Company (N FORWARD2018_4).

\section{Compliance with Ethical Standards}

Conflict of Interest The authors declare that they have no competing interests.

Consent to Participate All participants provided written informed consent.

Ethical Approval The study was approved by the Institutional Review Board of the Montpellier University Hospital (accreditation no. 198711).

Open Access This article is licensed under a Creative Commons Attribution 4.0 International License, which permits use, sharing, adaptation, distribution and reproduction in any medium or format, as long as you give appropriate credit to the original author(s) and the source, provide a link to the Creative Commons licence, and indicate if changes were made. The images or other third party material in this article are included in the article's Creative Commons licence, unless indicated otherwise in a credit line to the material. If material is not included in the article's Creative Commons licence and your intended use is not permitted by statutory regulation or exceeds the permitted use, you will need to obtain permission directly from the copyright holder. To view a copy of this licence, visit http://creativecommons.org/licenses/by/4.0/.

\section{References}

1. Enquête nationale périnatale Rapport 2016-Epopé Inserm. 2016. http://www.epope-inserm.fr/wp-content/uploads/2017/10/ ENP2016_rapport_complet.pdf. Accessed 31 Jan 2020.

2. Observatoire épidemiologique de la fertilité en France. 2015. http:// epidemiologie-france.aviesan.fr/epidemiologie-france/fiches/ epidemiological-observatory-for-fertility-in-france. Accessed 31 Jan 2020.

3. Le nouveau rapport d'activité de l'Agence de la biomédecine. 2017. $\mathrm{http} / / /$ www.agence-biomedecine.fr/Le-rapport-annuel-medical-et. Accessed 31 Jan 2020.

4. Noyes RW, Hertig AT, Rock J. Dating the endometrial biopsy. Fertil Steril. 1950;1:3-25.

5. Friedler S, Schenker JG, Herman A, Lewin A. The role of ultrasonography in the evaluation of endometrial receptivity following assisted reproductive treatments: a critical review. Hum Reprod Update. 1996;2(4):323-35.

6. Murray MJ, Meyer WR, Zaino RJ, Lessey BA, Novotny DB, Ireland $\mathrm{K}$, et al. A critical analysis of the accuracy, reproducibility, and clinical utility of histologic endometrial dating in fertile women. Fertil Steril. 2004;81(5):1333-43.

7. Haouzi D, Dechaud H, Assou S, De Vos J, Hamamah S. Insights into human endometrial receptivity from transcriptomic and proteomic data. Reprod BioMed Online. 2012;24(1):23-34.

8. Díaz-Gimeno P, Horcajadas JA, Martínez-Conejero JA, Esteban FJ, Alamá P, Pellicer A, et al. A genomic diagnostic tool for human endometrial receptivity based on the transcriptomic signature. Fertil Steril. 2011;95(1):50-60 60.e1-15.

9. Enciso M, Carrascosa JP, Sarasa J, Martínez-Ortiz PA, Munné S, Horcajadas JA, et al. Development of a new comprehensive and reliable endometrial receptivity map (ER map/ER grade) based on RT-qPCR gene expression analysis. Hum Reprod. 2018;33(2):220 8.

10. Haouzi D, Mahmoud K, Fourar M, Bendhaou K, Dechaud H, De Vos J, et al. Identification of new biomarkers of human endometrial receptivity in the natural cycle. Hum Reprod. 2009;24(1):198-205.

11. Haouzi D, Assou A, Mahmoud K, Tondeur S, Rème T, Hedon B, et al. Gene expression profile of the human endometrial receptivity: comparison between natural and stimulated cycles for the same patients. Hum Reprod. 2009;24(6):1436-45.

12. Haouzi D, Assou S, Dechanet C, Anahory T, Dechaud H, De Vos J, et al. Controlled ovarian hyperstimulation for in vitro fertilization alters endometrial receptivity in humans: protocol effects. Biol Reprod. 2010;82(4):679-86.

13. Haouzi D, Dechaud H, Assou S, Monzo C, de Vos J, Hamamah S. Transcriptome analysis reveals dialogues between human trophectoderm and endometrial cells during the implantation period. Hum Reprod. 2011;26(6):1440-9.

14. Bissonnette L, Drissennek L, Antoine Y, Tiers L, Hirtz C, Lehmann S, et al. Human S100A10 plays a crucial role in the acquisition of the endometrial receptivity phenotype. Cell Adhes Migr. 2016;10(3):282-98.

15. Haouzi D, Bissonnette L, Gala A, Assou S, Entezami F, Perrochia $\mathrm{H}$, et al. Endometrial receptivity profile in patients with premature progesterone elevation on the day of HCG administration. Biomed Res Int. 2014;2014:951937.

16. Hamamah S, Haouzi D (2011) Methods for assessing endometrium receptivity of a patient. Patent application number PCT/EP2011/ 058757

17. Polanski LT, Baumgarten MN, Quenby S, Brosens J, Campbell BK, Raine-Fenning NJ. What exactly do we mean by 'recurrent implantation failure'? A systematic review and opinion. Reprod BioMed Online. 2014;28(4):409-23. 
18. Tusher VG, Tibshirani R, Chu G. Significance analysis of microarrays applied to the ionizing radiation response. Proc Natl Acad Sci U S A. 2001;98(9):5116-21.

19. Rème T, Hose D, De Vos J, Vassal A, Poulain PO, Pantesco V, et al. A new method for class prediction based on signed-rank algorithms applied to Affymetrix microarray experiments. BMC Bioinformatics. 2008;11(9):16.

20. Drissennek L, Baron C, Brouillet S, Entezami F, Hamamah S, Delphine Haouzi D (2020) Endometrial miRNome profile according to the receptivity status and implantation failure. Hum Fertil

21. DeLong ER, DeLong DM, Clarke-Pearson DL. Comparing the areas under two or more correlated receiver operating characteristic curves: a nonparametric approach. Biometrics. 1988;44(3):837-45.

22. Kalma Y, Granot I, Gnainsky Y, Or Y, Czernobilsky B, Dekel N, et al. Endometrial biopsy-induced gene modulation: first evidence for the expression of bladder-transmembranal uroplakin $\mathrm{Ib}$ in human endometrium. Fertil Steril. 2009;4:1042-9 1049.e1-9.

23. Psychoyos A. Uterine receptivity for nidation. Ann N Y Acad Sci. 1986;476:36-42.

24. Navot D, Scott RT, Droesch K, Veeck LL, Liu HC, Rosenwaks Z. The window of embryo transfer and the efficiency of human conception in vitro. Fertil Steril. 1991;55(1):114-8.

25. Prapas Y, Prapas N, Jones EE, Duleba AJ, Olive DL, Chatziparasidou A, et al. The window for embryo transfer in oocyte donation cycles depends on the duration of progesterone therapy. Hum Reprod. 1998;13(3):720-3.

26. Lessey BA, Young SL. What exactly is endometrial receptivity? Fertil Steril. 2019;111(4):611-7.

27. Fatemi HM, Kyrou D, Bourgain C, Van den Abbeel E, Griesinger G, Devroey P. Cryopreserved-thawed human embryo transfer: spontaneous natural cycle is superior to human chorionic gonadotropin-induced natural cycle. Fertil Steril. 2010;94(6): 2054-8.

28. Xiao Z, Zhou X, Xu W, Yang J, Xie Q. Natural cycle is superior to hormone replacement therapy cycle for vitrificated-preserved frozen-thawed embryo transfer. Syst Biol Reprod Med. 2012;58(2): $107-12$.

29. Cardellicchio L, Reschini M, Paffoni A, Guarneri C, Restelli L, Somigliana E, et al. Frozen-thawed blastocyst transfer in natural cycle: feasibility in every day clinical practice. Arch Gynecol Obstet. 2017;295(6):1509-14.

30. Gelbaya TA, Nardo LG, Hunter HR, Fitzgerald CT, Horne G, Pease $\mathrm{EE}$, et al. Cryopreserved-thawed embryo transfer in natural or down-regulated hormonally controlled cycles: a retrospective study. Fertil Steril. 2006;85(3):603-9.

31. Mackens S, Santos-Ribeiro S, van de Vijver A, Racca A, Van Landuyt L, Tournaye H, et al. Frozen embryo transfer: a review on the optimal endometrial preparation and timing. Hum Reprod. 2017;13(11):2234-42.
32. van de Vijver A, Drakopoulos P, Polyzos NP, Van Landuyt L, Mackens S, Santos-Ribeiro S, et al. Vitrified-warmed blastocyst transfer on the 5 th or 7 th day of progesterone supplementation in an artificial cycle: a randomised controlled trial. Gynecol Endocrinol. 2017;33(10):783-6.

33. Devine K, Richter KS, Widra EA, McKeeby JL. Vitrified blastocyst transfer cycles with the use of only vaginal progesterone replacement with Endometrin have inferior ongoing pregnancy rates: results from the planned interim analysis of a three-arm randomized controlled non inferiority trial. Fertil Steril. 2018;109(2):266-75.

34. Ruiz-Alonso M, Blesa D, Díaz-Gimeno P, Gómez E, FernándezSánchez M, Carranza F, et al. The endometrial receptivity array for diagnosis and personalized embryo transfer as a treatment for patients with repeated implantation failure. Fertil Steril. 2013;100(3): 818-24.

35. Cho K, Tan S, Buckett W, Dahan MH. Intra-patient variability in the endometrial receptivity assay (ERA) test. J Assist Reprod Genet. 2018;35(5):929-30.

36. Tan J, Kan A, Hitkari J, Taylor B, Tallon N, Warraich G, et al. The role of the endometrial receptivity array (ERA) in patients who have failed euploid embryo transfers. J Assist Reprod Genet. 2018;35(4): 683-92.

37. Bassil R, Casper R, Samara N, Hsieh TB, Barzilay E, Orvieto R, et al. Does the endometrial receptivity array really provide personalized embryo transfer? J Assist Reprod Genet. 2018;35(7):1301-5.

38. Valde CT, Schutt A, Simon C. Implantation failure of endometrial origin: it is not pathology, but our failure to synchronize the developing embryo with a receptive endometrium. Fertil Steril. 2017;108(1):15-8.

39. Bashiri A, Halper KI, Orvieto R. Recurrent implantation failureupdate overview on etiology, diagnosis, treatment and future directions. Reprod Biol Endocrinol. 2018;16(1):121.

40. Haouzi D, Bissonnette L, Leture H, Entezami F, Chahine H, Olivennes F, Hamamah S Endometrial receptivity under hormone replacement therapy in oocyte-donation recipient patients: transcriptomic approach. Med Res Arch. 2015;2(1).

41. Ruiz-Alonso M, Galindo N, Pellicer A, Simón C. What a difference two days make: "personalized" embryo transfer (pET) paradigm: a case report and pilot study. Hum Reprod. 2014;29(6):1244-7.

42. Hashimoto T, Koizumi M, Doshida M, Toya M, Sagara E, Oka N, et al. Efficacy of the endometrial receptivity array for repeated implantation failure in Japan: a retrospective, two-centers study. Reprod Med Biol. 2017;16(3):290-6.

Publisher's Note Springer Nature remains neutral with regard to jurisdictional claims in published maps and institutional affiliations. 